\title{
(6) Advancing sports OPEN Access cardiology: blue sky thinking in Qatar
}

\author{
Bruce Hamilton, ${ }^{1}$ Mathew G Wilson, ${ }^{1}$ \\ Hakim Chalabi
}

\begin{abstract}
Qatar, with its wide blue sky and searing desert sands, is not a location that clinicians and academics might immediately associate with cutting-edge sports cardiology. However, Aspetar Hospital is a very active unit, addressing key questions of both international and regional importance. As highlighted in this issue of BJSM, there are a large number of academic and clinical challenges within sports cardiology. The importance of sports cardiology in high-level competitive sport is incontrovertible; it is critical in preparticipation screening, management of ongoing cardiac conditions in athletes and event management guidelines to name but a few. In recognition of this significant role, European, North American and other international cardiology groups are increasingly dedicating personnel and funding to this field. These organisations deserve thanks for their insight and support in defining this specialty.
\end{abstract}

\section{Evidence-based sports cardiology?}

Several fundamental sports cardiology issues are yet to achieve consensus opinion - even among opinion leaders. Where does this leave the practising sports medicine physician who is at the 'coalface' of sports cardiology? They are all too aware of the complexity of unresolved issues relating to preparticipation cardiac screening. They are the liaison between athlete, family, specialist and coaching staff in the event an athlete has to give up their lifelong dream (and financial security), based on the identification of pathological heart disease. Liaising among such parties requires that sports physicians understand the nature of these

${ }^{1}$ Department of Sports Medicine, ASPETAR, Qatar Orthopaedic and Sports Medicine Hospital, Doha, Qatar ${ }^{2}$ Assistant Chief Medical Officer, ASPETAR, Oatar Orthopaedic and Sports Medicine Hospital, Doha, Oatar

Correspondence to Dr Bruce Hamilton, ASPETAR,

Oatar Orthopaedic and Sports Medicine Hospital,

PO Box 29222, Doha, Oatar;

bruce.hamilton@aspetar.com complicated and at times, controversial cardiac issues. On the field of play and as the first to respond when an athlete collapses, sports physicians must also ensure appropriate first aid and emergency response facilities, as it is they who will ultimately be held accountable should a catastrophic event occur. Expanding knowledge in this field will permit evidence-based and challenging dialogue to occur with sports cardiologists, to the benefit of all parties. This latter point has recently been addressed by a team of experts, led by sports physician Dr Jonathon Drezner (http://blogs.bmj.com/bjsm/2012/03/09/ ekg-summit-in-seattle-successes-and-nextsteps/), in trying to provide appropriate educational resources for physicians, and it is incumbent on sports medicine physicians to grasp these learning opportunities.

\section{Aspetar Hospital}

Into this melting pot of issues, enter Aspetar, Qatar Orthopaedic and Sports Medicine Hospital. A sports medicine hospital built in 2007 to provide world class sports medicine services to athletes of the region. Unencumbered by historical biases and bureaucracy, Aspetar recognises the critical role cardiology plays in the comprehensive care of the athlete and has set about establishing a pre-eminent sports cardiology service. The goal was clear: minimise adverse cardiac events in athletes in Qatar and around the world. But if you were to establish a sports cardiology programme from scratch, where do you start? What do you actually want to do? What services and personnel do you include? How do you build a sustainable financial infrastructure? Given the current state of knowledge - what is best practice? Do you focus on screening, prevention or comprehensive reactive care? Do the same rules apply to athletes of Arabic origin as those of Caucasian origin, for whom most data exist? These were some of the challenges imposed upon those charged with establishing sports cardiology programme of excellence in this region.
This sports cardiology issue

Five years since its inception, Aspetar has collaborated with the BJSM to produce a cutting-edge sports cardiology themed issue. To reach this point we have invested heavily in high-quality sports medicine physicians, sports cardiologists, physiologists, nurses and administrative staff. We have recognised the need for high-quality research and have invested in all elements of this, as witnessed in this issue. We have developed auditable clinical pathways to ensure the highest level of care in the screening of athletes with Aspetar as a 'one stop shop', and we have invested in training and resourcing our staff to react appropriately should there be an adverse cardiac event. Finally, through the National Sports Medicine Programme, Aspetar ensures appropriate quality assurance and control procedures are in place in all sporting clubs and federations in Qatar, with particular attention paid to emergency response management in the event of a cardiac arrest. As a result, 5 years and 5000 athlete assessments later, we have a well grounded, high-quality and comprehensive sports cardiology programme, providing both an extensive clinical service and quality research database.

There is nothing easy about establishing a sports medicine centre in the Middle-East of the scale of Aspetar. Establishing a sports cardiology programme that provides world class care and research data does not happen spontaneously, particularly in the absence of identifiable best practice models. There remains much to be done. In coming years, Aspetar, in collaboration with leaders in the fields of sports medicine and cardiology - many of whom are featured in this issue - aims to address the challenging sports cardiology questions. Gathering reliable data on high level athletes, and collaborating with inspirational sports physicians and cardiologists from around the world, will ensure that athletes around the globe, as well as those of Gulf and Middle-Eastern origin, will benefit from the investment in sports medicine being made in Qatar. This is the new challenge that Aspetar is taking on.

Competing interests None.

Provenance and peer review Commissioned; internally peer reviewed.

Received 11 September 2012

Accepted 11 September 2012

Br J Sports Med 2012;46:i1

doi:10.1136/bjsports-2012-091777 